\title{
POLYARTERITIS NODOSA AND CONGENITAL PYLORIC HYPERTROPHY IN A 3-MONTH-OLD INFANT
}

\author{
BY \\ T. R. SAVAGE AND J. F. SMITH* \\ From Wanstead Hospital, Essex, and the Bernhard Baron Institute of Pathology, \\ London Hospital, E.1
}

(RECEIVED FOR PUBLICATION FEBRUARY 12, 1960)

\begin{abstract}
A case of polyarteritis nodosa in the first year of life with the clinical picture dominated by pyrexia, an initial upper respiratory element, episodes of tachycardia, and sudden death three weeks after the onset is described. The necropsy showed evidence of polyarteritis nodosa, myocarditis, and valvulitis as well as congenital pyloric hypertrophy. Clinical manifestations of the latter had preceded the final illness and the two diseases were not thought to be related.

The previous 19 cases reported in the first year of life are reviewed, and support is given for the concept that at this age a rash, pyrexia, upper respiratory manifestations, and progressive heart failure or sudden death are characteristic clinical manifestations and that the heart is often the site of the major lesions at necropsy.

The possible role of hypersensitivity is discussed. There was no evidence for it in the present case.
\end{abstract}

Polyarteritis nodosa is uncommon in infancy; references to 19 cases diagnosed as such in the first year of life have been found; there are a few others we have not been able to check (the cases of Rüger, 1944, and Wright, 1948). Of those checked all except the ones reported by De Pascale (1955) and Legros (1944) have proved fatal. These authors reported cases in which the main manifestations of the disease were in the skin, and it is possible that they were not dealing with examples of true polyarteritis nodosa but rather that group described as "allergic" vasculitis by McCombs, Patterson, and McMahon (1956) in which recovery is common. Many of the fatal cases had features indicating involvement of the skin, upper respiratory tract, and heart during life and maximal lesions in the coronary arteries at necropsy. In the infant reported in this paper such features were preceded by clinical symptoms of congenital pyloric hypertrophy which was also found at necropsy. Furthermore, a diffuse myocarditis and valvulitis similar to that of severe acute rheumatism formed part of the pathological picture.

\section{Case Report}

C.Q., a boy, had weighed $9 \mathrm{lb} .7 \mathrm{oz}$. at birth, the pregnancy and labour being normal. The father had had pulmonary tuberculosis which was reported cured

\footnotetext{
*Present address: University College Hospital Medical School University Street, London, W.C.1.
}

in 1946. There was no other family history of illness and none of allergy. The infant was breast fed and at the age of 9 weeks was admitted to hospital with a history of constipation and increasing vomiting after feeds for two weeks ; in the preceding four days every feed had been returned. A provisional diagnosis of hypertrophic pyloric stenosis was made. On examination the baby was well nourished and hydrated. There was visible peristalsis, but no pyloric tumour was palpated and there were no other physical signs. The vomiting ceased during administration of atropine methyl nitrate which was continued for several days.

In the absence of a pyloric tumour a diagnosis of feeding difficulty was made and he was discharged home after three days. The infant remained well and continued to gain weight until the age of 12 weeks, when a cough, purulent nasal and conjunctival discharge, and bilateral cervical lymph node enlargement developed. After five days he was again admitted to hospital ; pyrexia, mostly $100-103^{\circ} \mathrm{F}$., swinging at times, was present during the remainder of his life, a further two and a half weeks; the liver was palpable to two fingerbreadths.

Investigations. - No pathogens were isolated from nose and throat swabs or from faeces. The cerebrospinal fluid was clear ; cells $16 /$ c.mm. (50\% lymphocytes, $50 \%$ endothelial cells); protein $30 \mathrm{mg} . / 100 \mathrm{ml}$., sugar $55 \mathrm{mg} . / 100 \mathrm{ml}$. ; culture sterile. A blood count showed $\mathrm{Hb} 70 \%$; red cells 4.3 million; white cells 32,000 (neutrophils $85 \%$, eosinophils $5 \%$, lymphocytes $7 \%$, monocytes $3 \%$, reticulocytes $0.2 \%$ ) ; blood urea $35 \mathrm{mg} . / 100 \mathrm{ml}$. A blood culture showed a coagulasenegative staphylococcus (regarded as a contaminant). There was no agglutination with S. typhi, paratyphi, 


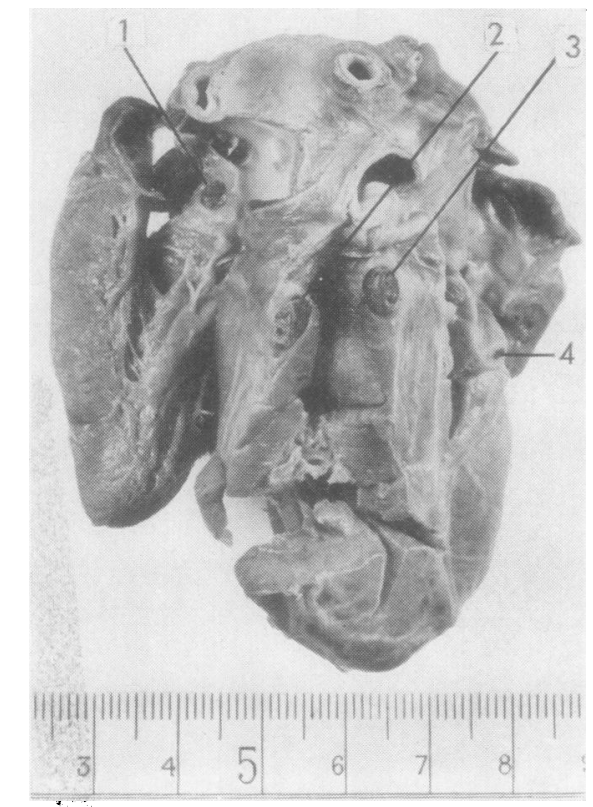

FIG. 1.-Fhatograph of heart. At 1, 2, and 3 aneurysms of the coronaries containing thrombus are seen in section. At 4 , a branch of the coronary is cut across as it runs down the surface of the ventricle; the thickened wall causes the vessel to stand out as a cord.

and typhi murium. A direct Coombs test was negative. The Mantoux test at 1:100 was negative. A report on iliac marrow (Dr. H. Caplin) read:

“... the main feature was intense granulocytosis with a preponderance of metamyelocytes and juvenile polymorphs. Nucleated red cells appeared scanty, the myeloid-erythroid ratio being approximately $30: 1$. The appearances were in keeping with a pronounced reaction to pyogenic infection."

Urine contained no protein or sugar ; a few leucocytes and epithelial cells were found in the deposit and a culture showed coagulase-negative staphylococcus (regarded as a contaminant).

Radiographs of the chest and the whole skeleton were normal. An electrocardiogram with a rate of 180 per min. approximately and with right axis deviation was interpreted as indicative of sinus tachycardia only.

Progress and Treatment.-A single injection of crystalline penicillin, 100,000 units, was given on admission, then chloramphenicol, $250 \mathrm{mg}$. by mouth six hourly for three days; and then streptomycin, $100 \mathrm{mg}$. 12 hourly, and penicillin, 100,000 units four hourly, both intramuscularly, for a further three days. Six days after this, terramycin, $70 \mathrm{mg}$. six hourly by mouth, was given for 48 hours. None of these drugs had any effect on the pyrexia. The only other drug given was atropine methyl nitrate, 3 drops before feeds during the first five days of this second admission.
A sudaminal type of rash appeared on the upper limbs three days after admission and was present $\overrightarrow{\bar{\omega}}$ intermittently during the rest of the illness on all limbs. The liver remained palpable, the apical pulse rate was 160-180 on two occasions, the complexion $\frac{\bar{C}}{\bar{D}}$ was sallow but without jaundice. Feeding was satis- $\frac{\rho}{T}$ factory and stools were normal. Dr. Kenneth Tallerman kindly agreed to accept responsibility for the care of the baby and he was transferred to the London Hospital where he suddenly collapsed and $\vec{\circ}$ died 60 hours after admission. In that time the $\overrightarrow{\vec{\omega}}$ laboratory findings remained unchanged; a chest film was normal and a blood culture sterile.

Necropsy Report.-The findings may be summar-? ized as acute heart failure, myocarditis, polyarteritis $\vec{\omega}$ rodosa, and congenital pyloric hypertrophy. Body $\rightarrow$ weight $15 \frac{1}{2} \mathrm{lb}$.

Heart $(2$ oz.).-Scattered nodular aneurysms of the circumflex branch of the left and terminal part of $\frac{}{\partial}$ the right coronary artery were present; they had abluish-red, projecting surface and on section contained $\subseteq$ recent thrombus (Fig. 1), the latter extending for a few centimetres on either side of the dilatation. The wall $\vec{\omega}$ of the right coronary artery was slightly thickened (up to $0.05 \mathrm{~cm}$.) proximal to the thrombus. Most of the free margin of the mitral valve and a segment (1.5 $\mathrm{cm}$. long) of the tricuspid valve were slightly

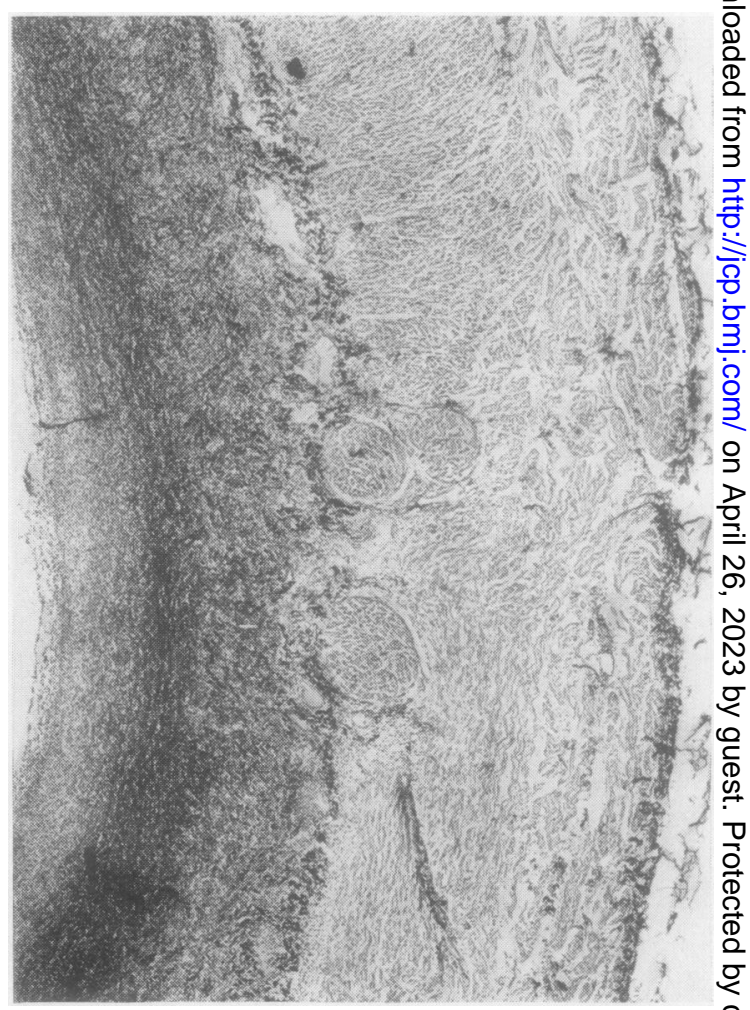

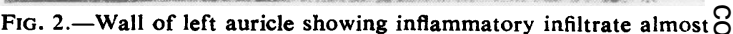
as thick as the muscle of the wall. Verhoeff's elastic stain, $\times 26$. 


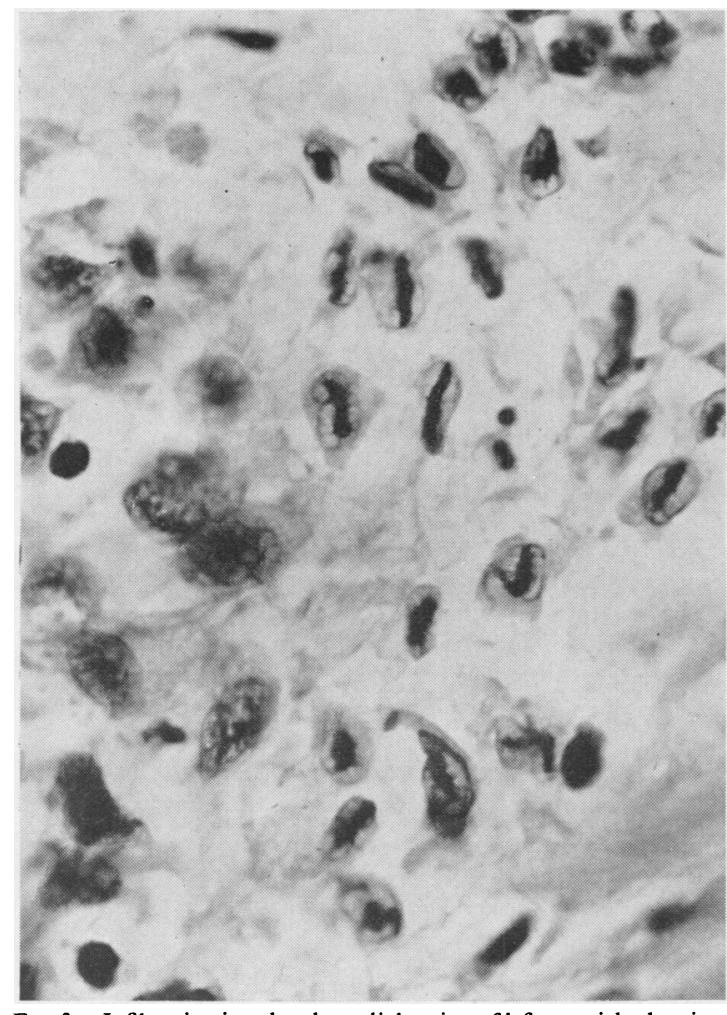

FIG. 3.-Infiltration in subendocardial region of left ventricle showing numerous Anitschkow myocytes. Haematoxylin and eosin, $\times 830$.

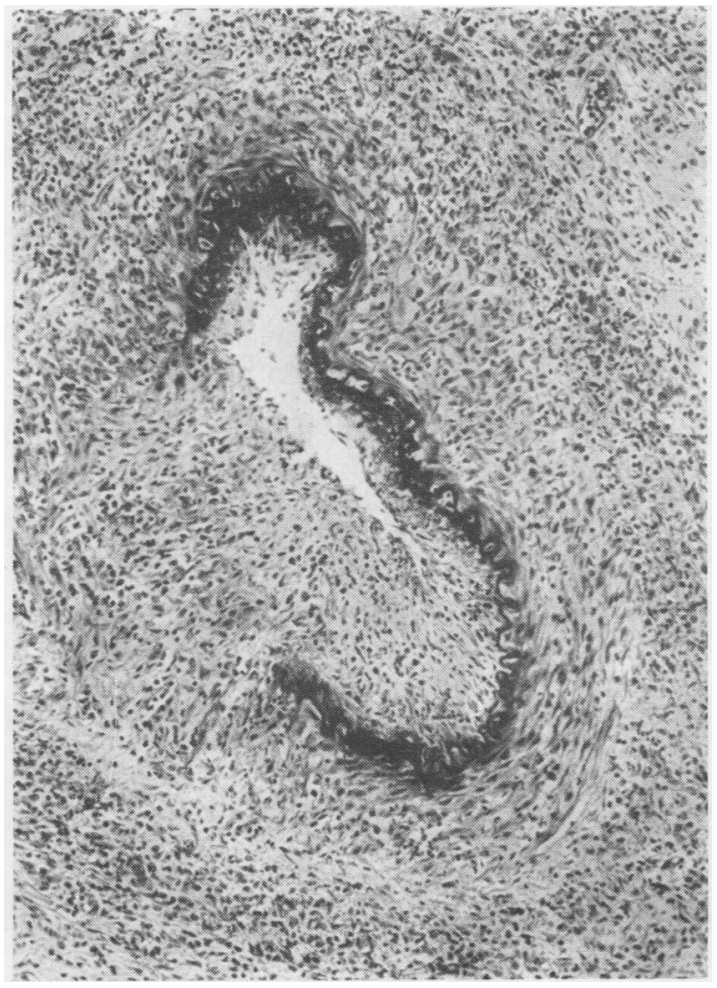

FIG. 5.-Artery in splenic capsule with conspicuous inflammatory infiltration and focal destruction of prominent internal elastic lamella and muscle of media. Haematoxylin and eosin, 110.

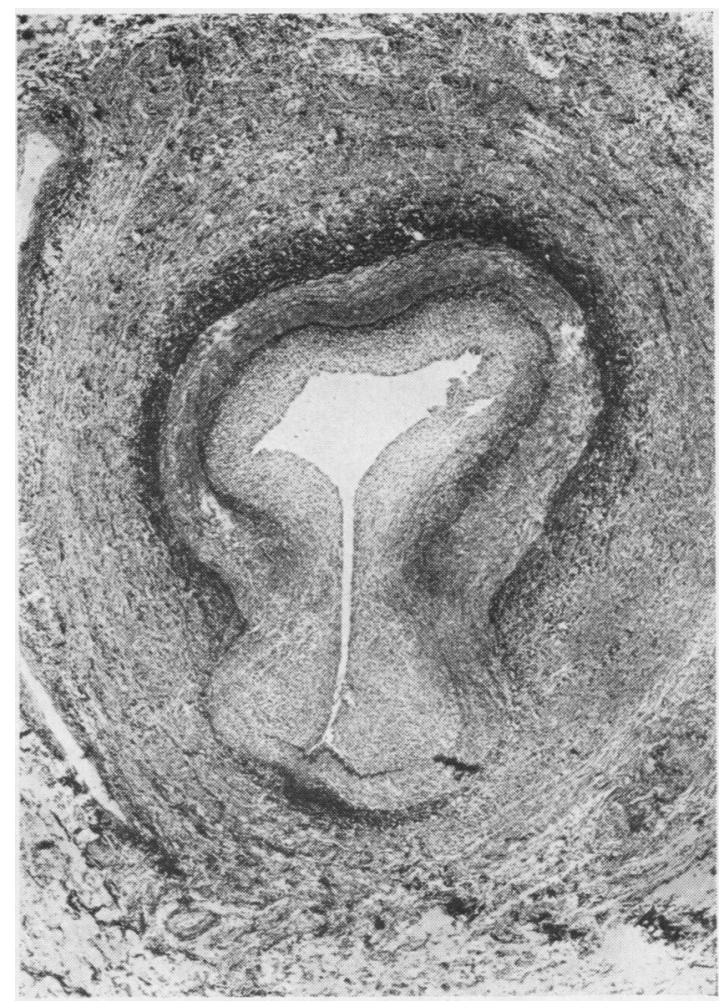

FIG. 4.-Right coronary artery proximal to thrombus with considerable inflammatory thickening of the intima and focal destruction of elastic and muscle of media. Verhoeff's elastic stain, $\times 23$.

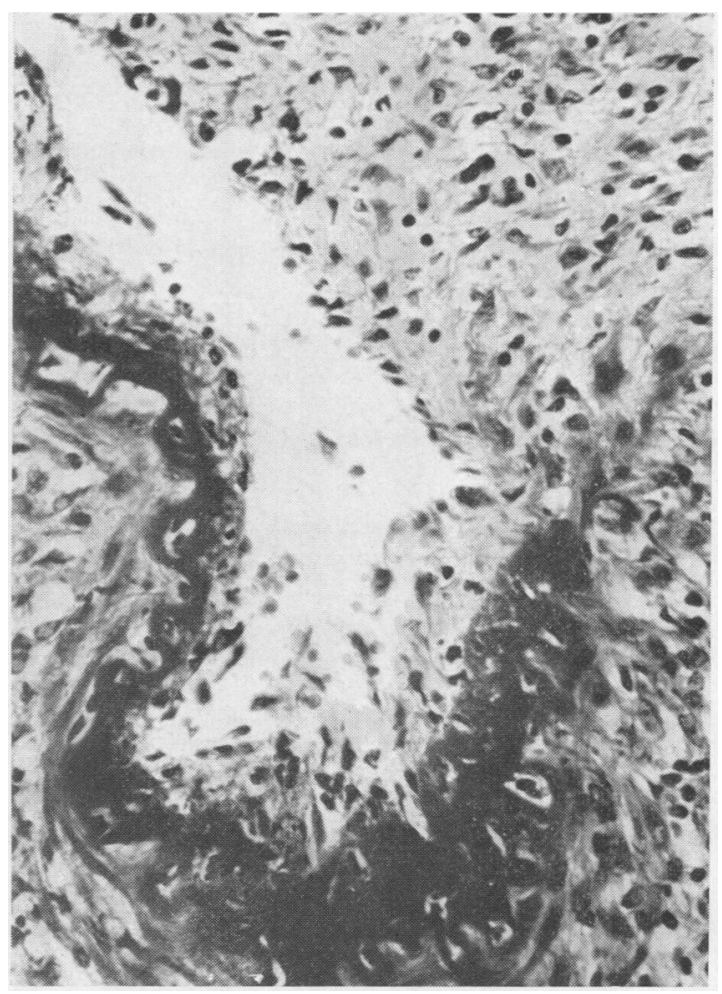

FIG. 6.-One segment of artery of Fig. 5. Mononuclear cells predominate in the infiltrate. There are a few smudges of fibrinoid material (black) just internal to the elastic lamella, especially in the concavity of the segment. Haematoxylin and eosin, $\times 330$. 
thickened; pinhead haematomas were also present in these, one at the root of the tricuspid, one near its free edge, and $a$ third near the free edge of the mitral. The myocardium was firm and greyish brown with a slightly streaky appearance on the fresh cut surface in some areas of the left ventricle.

Other Organs.-The kidneys were congested; the lungs slightly congested. The muscle of the pyloric end of the stomach was conspicuously thickened $(0.5$ $\mathrm{cm}$.), but there was no stenosis.

Microscopical Examination.-This was undertaken on tissues from all the cardiac chambers, mitral and tricuspid valves, coronary arteries, liver, spleen, kidneys, alimentary tract, endocrines, several lymphatic glands, the submandibular salivary gland, both lungs, cerebellum, pons, basal ganglia, and left temporal lobe.

There was an extensive inflammatory infiltration in the heart which was maximal in and around the walls of the larger coronary arteries, in the endocardium of the left auricle (Fig. 2), and at the root of the mitral valve but was also conspicuous in patches in the subendocardial region of the left ventricle and to a less extent in the right auricle and ventricle. The walls of small coronary arteries were in general spared, but the endothelium of a few veins was infiltrated. The mitral valve was infiltrated from root to free margin but the tricuspid only at the root. Histiocytes and lymphocytes were very numerous in all sites; polymorphonuclear leucocytes abundant in most sites, numerous eosinophil leucocytes in only a few sites. In some areas there were scattered fibroblasts and some delicate, newly formed collagen fibrils. In the subendocardial region of the left ventricle Anitschkow myocytes were abundant (Fig. 3) and in several areas some were large with twisted hyperchromatic nuclei reminiscent of the cells found in Aschoff bodies; an occasional histiocyte was seen in mitosis. In the myocardium the infiltrate was perivascular for the most part, but a few small groups of cells were found between muscle fibres. In some areas the connective tissue at the site of infiltration stained poorly and in a few there was slight fragmentation, but fibrinoid change was only seen in one small area in the epicardium anterior to the interventricular septum. The infiltration was thus somewhat like that of acute rheumatism, but the cellular types were even more pleomorphic than in that condition.

In the larger coronary arteries the infiltration was often associated with partial destruction of the elastic lamellae and of muscle of the media; in some the thickening of the intima was very conspicuous (Fig. 4), but in none was there fibrinoid material in the wall. At the sites of aneurysms there was great focal thinning of the media and recent thrombus in the lumen.

A similar arteritis involved one vessel in the splenic capsule (Figs. 5 and 6) and two in the capsules of the suprarenals. In the splenic one there was focal destruction of the internal elastic lamella, patches of fibrinoid internal to it, and conspicuous cellular thickening of the intima; in the suprarenal vessels the internal elastic lamellae had a fibrinoid layer closely apposed to their inner aspect, but appeared to be intact. The adventitia was conspicuously thickened by cellular infiltration, and this encroached in places into the media.

In the section of the upper lobe of the left lung focal infiltrations of the walls of large pulmonary arteries and veins mainly by polymorphonuclear $\vec{\circ}$ leucocytes and lymphocytes, were associated with intimal intraluminal projections and focal partial destruction of the elastic of the wall in the arteries. In this and also in the section of the right upper lobe chronic inflammation of the walls of a few bronchi was present. In the kidney there was adventitial infiltration of an artery in the lower cortex.

The only other histological abnormalities were inflammation of the leptomeninges, of the capsule and periportal regions of the liver, of the sinuses of the lymph glands and around a few small veins in the thyroid.

Reasons for Diagnosis.-Destructive and inflammatory lesions of large branches of the coronary arteries and of smaller ones in the suprarenal and splenic capsules were present, the former being accompanied by aneurysm formation and thrombosis, the latter showing focal fibrinoid change. These justify a diagnosis of polyarteritis nodosa. The presence of slighter but definite lesions in pulmonary arteries and veins makes its nosological place uncertain if the classification of Zeek (1952) is used. This and the significance of the myocarditis will be considered in the discussion. The congenital pyloric hypertrophy is regarded as a coincidental and unrelated finding.

\section{Discussion}

It is convenient to summarize five of the cases reported of polyarteritis nodosa occurring in infancy, and to give very brief details of others, in order to illustrate the variety of clinical and pathological manifestations before considering the significance of the peculiar features of the present case.

Krzyszkowski (1899) described a $2 \frac{1}{2}$-month-old baby with an indefinite skin rash, slight oedema of the eyelids and extremities, and pyrexia. A clinical diagnosis of scarlet fever or syphilis was made and death occurred 11 days after the onset. Necropsy showed small thrombosed aneurysms of the coronary arteries which were characteristic of polyarteritis nodosa on microscopic examination. Although bronchopneumonia, enteritis, and nephritis were also described, arterial lesions were not found outside the heart.

Scott and Rotondo (1944) described a 9-monthold female in whom a rash developed after the fifth dose of sulphathiazole $(0.5 \mathrm{~g}$. every four hours was given for pharyngitis with pyrexia). 
Pyuria and oedema of the face and limbs were later manifestations, while radiographs indicated cardiac hypertrophy and an inflammatory process at the base of the right lung. The child died suddenly five weeks after the initial symptoms at a time when clinical improvement was thought to be occurring. At necropsy, thrombosed aneurysms were present in the coronary arteries of the enlarged heart. Microscopy indicated these to be due to polyarteritis nodosa, which was also found in the kidneys, liver, and uterus.

Fager, Bigler, and Simonds (1951) reported a 4-month-old male infant who had an illness resembling virus pneumonia initially. Pyrexia was persistent despite treatment with penicillin, streptomycin, aureomycin, and sulphonamides. There was no rash, but in the terminal stages the respiratory and cardiac symptoms and signs suggested a myocarditis. At necropsy thrombosed dilated coronary arteries in the enlarged heart were recanalized and there were infarcts in spleen and kidneys; the left renal artery was thrombosed. Microscopy indicated polyarteritis nodosa in these organs and focal myocarditis.

Adelson (1951) described a male infant in whom the illness began at the age of 15 weeks with pyrexia, slight cough, and moderate cervical gland enlargement which persisted throughout an illness of 22 days. Sensitivity to penicillin, which had been used initially, was strongly suggested by the development of a rash following its administration on two separate occasions. The final episode of screaming, gasping, and cyanosis in which death occurred was sudden and unexpected. At necropsy there was conspicuous nodular "beading," narrowed lumina, and aneurysmal dilatation of coronary arteries "with thrombosis in the circumflex. The histology showed characteristic changes of polyarteritis in the heart and other organs.

Martelle (1955) did not classify his case of "coronary thrombosis in a 5-month-old infant" as an example of polyarteritis although the clinical and pathological features were similar to those of many recorded examples. The illness started at 4 months with fever, vomiting, loose stools, and a dry skin. The baby was treated with penicillin and appeared to recover satisfactorily. Two weeks later there was a two-day episode of irritability, anorexia, and vomiting without pyrexia. The baby recovered, but when 5 months old he had another episode in which sudden death occurred. At necropsy the enlarged heart showed aneurysmal dilatation and thrombosis of all the coronary arteries. Microscopical examination showed severe arteritis, involving chiefly the coronaries, but also the aorta and extensive myocarditis and endocarditis. The latter affected the left auricle but not apparently the valves; and although distinct Aschoff bodies were not present "the grouping of some of the mononuclear cells was suggestive, as well as their individual appearance." Areas of muscle necrosis were not thought sufficient to account for the myocarditis.

Of the other cases recorded it is worth while noting that marked pulmonary arteritis was a feature in the newborn infant described by Elwood (1955) and in the case of Liban, Shamir, and Schorr (1954).

Johansmann and Zeek (1954) described a 7-dayold baby in whom scattered arterial lesions were thought to correspond to that type of polyarteritis nodosa which she refers to as the classical one and in which hypertension may be an important pathogenic factor. This example had also a thrombosed aneurysm of a ductus arteriosus and extensive infarction of the right kidney. The latter was associated with thrombosis of a branch of the renal artery but not with arteritis.

The infant described by Bohman (1954) was unique in that the illness began after pertussis prophylaxis had been given, but the course suggested that a septic process, starting in subcutaneous abscesses, was more important in pathogenesis. In Thinnes' (1924) case the only symptom was abdominal pain four hours before death and at necropsy histological evidence of polyarteritis was present in the heart and kidneys. The two cases described by Wilmer (1945) occurred in the first month of life; both had a severe illness, one with skin lesions, and extensive lesions were found in the heart, kidneys, uterus, and other viscera. The 9-month female infant reported by Diaz-Rivera and Miller (1946) fell into the skin, upper respiratory, cardiac group, as did that of Sinclair and Nitsch (1949). In both of these sulphonamide drugs had been given, in the latter penicillin also.

The case reported by Korb (1957) had a clinical picture suggestive of nephritis and sepsis, and polyarteritis was only diagnosed on microscopic examination; that of Rose (1957) had aneurysms of branches of the aorta as well as coronary involvement.

Munro-Faure (1959) has reported an infant with marked coronary involvement and reviewed a series which overlaps extensively with the one considered here. Her paper is called "Necrotizing Arteritis of the Coronary Vessels." She has therefore not included examples of poly- 
arteritis in which the coronaries were not involved and has included two cases in which the authors, Crocker, Sobin, and Thomas (1957), considered the diagnosis of polyarteritis nodosa but were not prepared to make it. Before reading her paper we had noted the frequent involvement of the skin in life and of the coronary arteries at necropsy in this group.

In her somewhat more selected series this point comes out clearly, and we would support the contention that there is a group of cases in infants in which an arteritis is maximal in the coronaries and the illness is frequently characterized by fleeting macular skin eruptions, fever, leucocytosis, conjunctivitis, and pharyngitis, and in which death occurs either as a result of progressive heart failure or suddenly and unexpectedly. Cervical adenitis and cough were common but not quite so frequent in this group. If the features of the pyloric hypertrophy are regarded as a separate process the present case obviously illustrates many features of what might be called the upper respiratory, skin, cardiac group. Of the 18 cases discussed here, eight fall easily into this group and lesions of the coronaries were present to some extent in $16(88 \%)$. In the 111 cases of polyarteritis recently reviewed by Rose (1957), which included mainly adult material, $48 \%$ had coronary lesions. In none of these infantile cases was there the prolonged uraemia, hypertension, or peripheral neuritis which characterize an appreciable number of adult cases.

There remain for consideration the significance of the myocarditis and valvulitis in our case and the possible role of hypersensitivity in the pathogenesis. The myocarditis was probably responsible for the episodes of tachycardia during life, and a similar correlation has been noted in other infantile cases. Extensive myocarditis, however, is uncommon in this age group, endocarditis was only noted in the case of Martelle (1955), while the valvulitis of the present case was unique.

The presence of these features suggests that our case might be classified as an example of rheumatic carditis with polyarteritis. Such have been described in the past by Neale and Whitfield 1934) and Friedberg and Gross (1934) in children and adults but not in infants. The only infant which could be so classified in addition to our own is that of Martelle (1955). Clinical manifestations of rheumatism outside the heart and the association with a streptococcal infection which have been a feature of rheumatic carditis with polyarteritis were lacking in the infantile cases. At this age, furthermore, acute rheumatism is almost unknown.
We do not therefore wish to press this point $\underline{\text { 을 }}$ nor the interwoven one of the possibility of a $\overrightarrow{\vec{F}}$ hypersensitive reaction in the pathogenesis. This $\stackrel{5}{+}$ would involve a detailed discussion of present-day views on the role of hypersensitivity in polyarteritis which was originally emphasized so $\frac{\text { क }}{\vec{D}}$ clearly by Rich (1947) and more sharply defined $\stackrel{\odot}{\circ}$ in Zeek's (1952) concept of "hypersensitivity के angeiitis." Our case does not fit into this category $\vec{\circ}$ and the concept has not been confirmed in the survey of Rose and Spencer (1957). And although $\vec{\omega}$ the present example yields no clear evidence of a hypersensitive reaction to any of the drugs ? given, one is left with the impression that the $\vec{\omega}$ morphological changes are more easily explained $\stackrel{t}{\rightarrow}$ in terms of such a process than in any other way. 응 That antibodies can form in the early months of life is indicated by the investigations of Sako 옥 (1947) and Sako, Treuting, Witt, and Nichamin $\vec{c}$ (1945), and the case of Adelson (1951) suggests strongly that anaphylactic hypersensitivity can

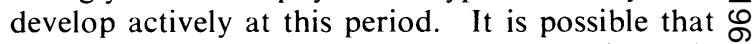
in these early months there may be considerable 0 variation in the speed and degree of loss of the immunological tolerance of foetal life and occasional unusual manifestations of reaction to $\bar{\partial}$ antigenic stimulation.

We are indebted to Dr. Eric Frankel and Dr. Kenneth Tallerman for allowing us to describe a case $\mathrm{O}$ under their care, and to Professor Dorothy Russell for advice about the pathology.

\section{REFERENCES}

Adelson, L. (1951). J. Pediat., 39, 346.

Bohman, M. (1954). Acta paediat. (Uppsala), 43, 374

Crocker, D. W., Sobin, S., and Thomas, W. C. (1957). Amer. J. Path 33, 819 .

De Pascale, A. (1955). Minerva pediat. (Toriso), T, 1217

Diaz-Rivera, R. S., and Miller, A. J. (1946). Ann. intern. Med., 24,

Elwood, J. S. (1955). A.M.A. Arch. Path., 60, 179

Fager, D. B., Bigler, J. A., and Simonds, J. P. (1951). J. Pediat. 음 39,65 .

Friedberg, C. K.. and Gross, L. (1934). Arch. intern. Med., 54, 170. Johansmann, R. J., and Zeek, P. (1954). A.M.A. Arch. Path.. 58, 207. O Korb, G. (1957). Z. ges. inn. Med., 12, 605.

Krzyszkowski, J. (1899). Quoted by Wilmer (1945).

Legros, J. (1944). Arch. franc. Pédiat., 2, 112. ban, E., Shamir, Z., and Schorr, S. (1954). Amer. J. Dis. Child., W 88, 210.

Martelle, R. R. (1955). J. Pediat., 46, 322

McCombs, R. P., Patterson, J. F., and McMahon, H. E. (1956). New Engl. J. Med., 255, 251.

Munro-Faure, H. (1959). Pediatrics, 23, 914.

Neale, A. V., and Whitfield, A. G. W. (1934). Brit. med. J., 2, 104.

Rich, A. R. (1947). Harvey Lect., 1946-1947, 42, 106.

Rose, G. A. (1957). Brit. med. J., 2, 1148.

Rose, G. A. (1957). Brit.med. J., 2, 1148.

Rüger, I. (1944). Kinderärztl. Prax., 15, 99.

Sako, W. (1947). J. Pediat., 30, 29.

Treuting, W. L., Witt, D. B., and Nichamin, S. J. (1945). Amer. med. Ass., 127, 379

Scott, E. P., and Rotondo, C. C. (1944). J. Pediat., 25, 306. Sinclair, W., and Nitsch, E. (1949). Amer. Heart J., 38, 898.

Thinnes, P. (1924). Frankfurt Z. Path., 30, 104.

Wilmer, H. A (1945). Bull. Johns Hopk. Hosp. 77, 275.

Wright, 1. S. (1948). Quoted by Korb in Vascular Diseases in Clinical Practice. Year Book Publishers, Chicago.

Zeek, P. M. (1952). Amer. J. clin. Path., 22, 777. 